\title{
A FULLY ANISOTROPIC MECHANISM FOR FORMATION OF TRAPPED SURFACES IN VACUUM
}

\author{
SERGIU KLAINERMAN, JONATHAN LUK, AND IGOR RODNIANSKI
}

\begin{abstract}
We present a new, fully anisotropic, criterion for formation of trapped surfaces in vacuum. More precisely we provide local conditions on null data, concentrated in a neighborhood of a short null geodesic segment (possibly flat in all other directions) whose future development contains a trapped surface. This extends considerably the previous result of Christodoulou [2] which required instead a uniform condition along all null geodesic generators. To obtain our result we combine Christodoulou's mechanism for the formation of a trapped surface with a new deformation process which takes place along incoming null hypersurfaces.
\end{abstract}

\section{InTRODUCTION}

According to the celebrated incompleteness result of Penrose, the future Cauchy development of a non-compact initial data set of the Einstein vacuum ${ }^{1}$ equations,

$$
\operatorname{Ric}(g)=0
$$

which contains a trapped surface, must be incomplete. Thus, in a sense, the fundamental issue of formation of spacetime singularities in gravitational collapse is reduced to the somewhat more tangible problem of formation of trapped surfaces. This, on the other hand, is still a highly non-trivial problem. Indeed, the expansions of both null geodesic congruences generated by a compact, trapped surface $S$ is required, by definition, to be negative at every point on $S$. To show that such surfaces can form in evolution, starting with regular initial data sets which contain no trapped surfaces, requires a deep understanding of the dynamics of the Einstein equations. It is for this reason that the problem has remained open for more than forty years, in the wake of Penrose's result, until the recent breakthrough of Christodoulou. In [2] he was able to identify an open set of regular ${ }^{2}$ initial conditions, on a finite outgoing null hypersurface, with trivial data on an incoming null hypersurface, whose future development must contain a trapped surface. The main condition in Christodoulou's result is that the data verify a uniform lower bound condition, with respect to all short, null geodesic generators of the outgoing initial null hypersurface. The goal of this paper is to significantly relax this

1991 Mathematics Subject Classification. 35Q76.

\footnotetext{
${ }^{1}$ The result of Penrose applies in fact to the more general Einstein-matter equations satisfying the null energy condition, but we restrict our considerations here to the vacuum case.

${ }^{2}$ Smooth and free of trapped surfaces.
} 
uniform condition by showing that a trapped surface forms even if the local null outgoing data is only concentrated in a neighborhood of a short null geodesic segment (possible flat in all other directions).

We recall that Christodoulou's proof in [2] rests on two main ingredients:

(1) A semi-global existence result for the characteristic initial value problem with large initial data $^{3}$ measured relative to a small parameter $\delta>0$. The precise dependence on $\delta$, which Christodoulou calls the short pulse method, was subsequently relaxed in [5], [6]; see also [8]. In all these results the data on the incoming null hypersurface is assumed to be flat. This restriction has been recently removed in [7].

The semi-global result allows one to construct the future development of the initial data, together with a double null foliation ${ }^{4}(u, \underline{u}), 0 \leq u \leq u_{*}, 0 \leq \underline{u} \leq \underline{u}_{*}$, and full control on all the geometric quantities associated to it.

(2) An amplification mechanism for the integrals $\int_{\gamma}|\hat{\chi}|^{2}$ along outgoing null geodesic segments $\gamma$, (with $\hat{\chi}$ denoting the outgoing null shear). This mechanism, which requires the estimates obtained in the constructive step (1), combines with a uniform lower bound assumption of these integrals on the initial null hypersurface, and leads to the formation of a trapped surface. It is important to note that all trapped surfaces found in this fashion belong to the concentric spheres generated by the double null foliation $(u, \underline{u})$, i.e. are of the form $S=\left\{u=u_{1}, \underline{u}=\underline{u}_{1}\right\}$, for some $0<u_{1} \leq u_{*}, 0<\underline{u}_{1} \leq \underline{u}_{*}$.

The new result we present in this paper relies heavily on the hard part of the above results, i.e. the construction of the spacetime in (1). We modify however part (2) by combining Christodoulou's argument with a new deformation argument along the incoming null hypersurfaces $\{\underline{u}=$ const $\}$. This allows us to dramatically weaken his uniform condition merely to a localized condition in a neighborhood of a null geodesic of $\{u=0\}$. The deformation is determined by solving a simple elliptic inequality on the standard sphere $S_{0,0}=\{\underline{u}=0\} \cap\{u=0\}$, see (20). We note that the trapped surface we find by our argument does not belong any longer to the double null foliation constructed in step (1).

1.1. Geometry of a double null foliation. As in [5] we consider a region $\mathcal{D}=\mathcal{D}\left(u_{*}, \underline{u}_{*}\right)$ of a vacuum spacetime $(M, g)$,

$$
\operatorname{Ric}(g)=0,
$$

spanned by a double null foliation generated by the optical functions $(u, \underline{u})$ increasing towards the future $^{5}$, where $0 \leq u \leq u_{*}$ and $0 \leq \underline{u} \leq \underline{u}_{*}$ (see Figure 1). These spacetimes will be constructed via solving a characteristic initial value problem.

\footnotetext{
${ }^{3}$ On the outgoing null hypersurface. The incoming data is flat.

${ }^{4}$ Such that the initial configuration is given by the incoming $\{\underline{u}=0\}$ and outgoing $\{u=0\}$ initial null hypersurfaces.

${ }^{5}$ These can be compared to the optical functions $u=\frac{t-r+1}{2}, \underline{u}=\frac{t+r-1}{2}$ in Minkowski spacetime.
} 

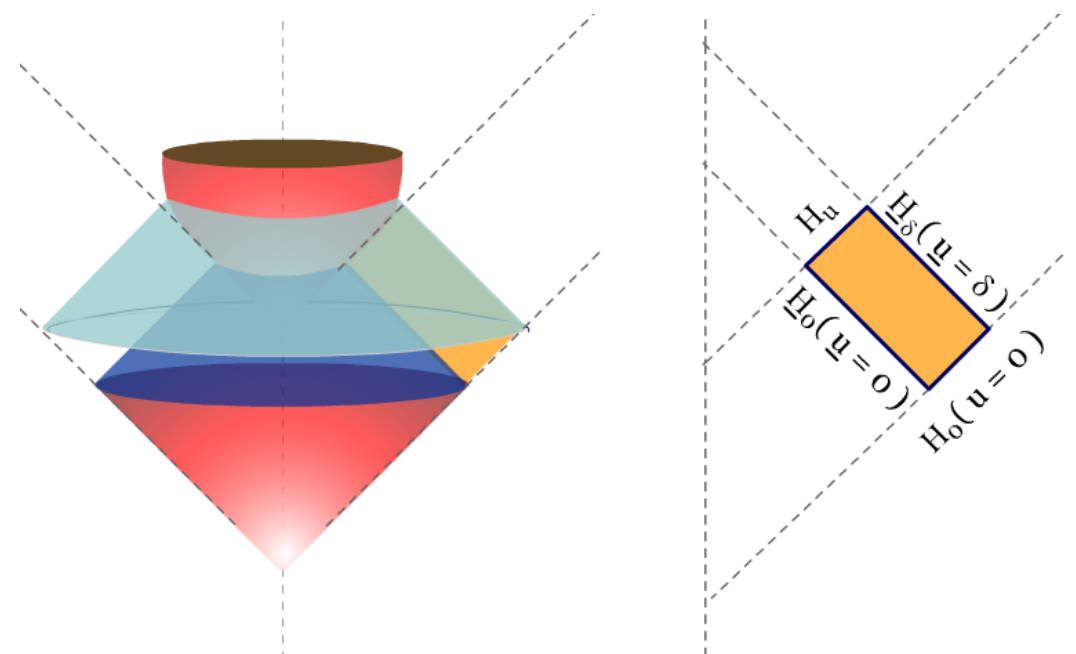

The shaded region on the right represents the domain $\mathcal{D}\left(u_{*}, \underline{u}_{*}\right), \underline{u}_{*}=\delta$. The same picture is represented on the left, by emphasizing that all points in the diagram on the right are in fact 2 -surfaces.

We denote by $H_{u}$ the outgoing null hypersurfaces generated by the level surfaces of $u$ and by $\underline{H}_{u}$ the incoming null hypersurfaces generated level hypersurfaces of $\underline{u}$. We write $S_{u, \underline{u}}=H_{u} \cap \underline{H}_{\underline{u}}$ and denote by $H_{u}^{\left(\underline{u}_{1}, \underline{u}_{2}\right)}$ and $\underline{H}_{\underline{u}}^{\left(u_{1}, u_{2}\right)}$ the regions of these null hypersurfaces defined by $\underline{u}_{1} \leq \underline{u} \leq \underline{u}_{2}$ and respectively $u_{1} \leq u \leq u_{2}$. Let $L=-2 g^{\alpha \beta} \partial_{\alpha} u \partial_{\beta}, \underline{L}=-2 g^{\alpha \beta} \partial_{\alpha} \underline{u} \partial_{\beta}$, be the geodesic vectorfields associated to the two foliations and define,

$$
g(L, \underline{L}):=-2 \Omega^{-2}=4 g^{\alpha \beta} \partial_{\alpha} u \partial_{\beta} \underline{u} .
$$

As is well known, the space-time slab $\mathcal{D}\left(u_{*}, \underline{u}_{*}\right)$ is completely determined (for small values of $u_{*}, \underline{u}_{*}$ ) by data along the null hypersurfaces $H_{0}, \underline{H}_{0}$ corresponding to $\underline{u}=0$ and $u=0$ respectively. We assume that $\Omega=1$ along $H_{0}$ and $\underline{H}_{0}$, i.e.,

$$
\begin{array}{ll}
\Omega(0, \underline{u})=1, & 0 \leq \underline{u} \leq \underline{u}_{*}, \\
\Omega(u, 0)=1, & 0 \leq u \leq u_{*} .
\end{array}
$$

We denote by $r=r(u, \underline{u})$ the area-radius of the 2 -surface $S=S_{u, \underline{u}}$, i.e. $\left|S_{u, \underline{u}}\right|=4 \pi r(u, \underline{u})^{2}$. In this paper, we assume $r(0,0)=1 .{ }^{6}$

Throughout this paper we work with the normalized null pair $\left(e_{3}, e_{4}\right)$ defined by

$$
e_{3}=\Omega \underline{L}, \quad e_{4}=\Omega L
$$

which satisfy

$$
g\left(e_{3}, e_{4}\right)=-2 .
$$

Given a 2-surfaces $S_{u, \underline{u}}$ and $\left(e_{a}\right)_{a=1,2}$ an arbitrary frame tangent to it we define the Ricci coefficients,

$$
\Gamma_{(\lambda)(\mu)(\nu)}=g\left(e_{(\lambda)}, D_{e_{(\nu)}} e_{(\mu)}\right), \quad \lambda, \mu, \nu=1,2,3,4 .
$$

\footnotetext{
${ }^{6}$ General values for $r(0,0)$ can be recovered from this special case via a simple rescaling argument.
} 
These coefficients are completely determined by the following components,

$$
\begin{array}{ll}
\chi_{a b}=g\left(D_{a} e_{4}, e_{b}\right), & \underline{\chi}_{a b}=g\left(D_{a} e_{3}, e_{b}\right), \\
\eta_{a}=-\frac{1}{2} g\left(D_{3} e_{a}, e_{4}\right), & \underline{\eta}_{a}=-\frac{1}{2} g\left(D_{4} e_{a}, e_{3}\right) \\
\omega=-\frac{1}{4} g\left(D_{4} e_{3}, e_{4}\right), \quad \underline{\omega}=-\frac{1}{4} g\left(D_{3} e_{4}, e_{3}\right), \\
\zeta_{a}=\frac{1}{2} g\left(D_{a} e_{4}, e_{3}\right)
\end{array}
$$

where $D_{a}=D_{e_{(a)}}$. We also introduce the null curvature components,

$$
\begin{array}{rlrl}
\alpha_{a b} & =R\left(e_{a}, e_{4}, e_{b}, e_{4}\right), \quad \underline{\alpha}_{a b} & =R\left(e_{a}, e_{3}, e_{b}, e_{3}\right), \\
\beta_{a} & =\frac{1}{2} R\left(e_{a}, e_{4}, e_{3}, e_{4}\right), \quad \underline{\beta}_{a}=\frac{1}{2} R\left(e_{a}, e_{3}, e_{3}, e_{4}\right), \\
\rho & =\frac{1}{4} R\left(L e_{4}, e_{3}, e_{4}, e_{3}\right), \quad \sigma=\frac{1}{4} * R\left(e_{4}, e_{3}, e_{4}, e_{3}\right) .
\end{array}
$$

Here ${ }^{*} R$ denotes the Hodge dual of $R$. We denote by $\nabla$ the induced covariant derivative operator on $S_{u, \underline{u}}$ and by $\nabla_{3}, \nabla_{4}$ the projections to $S_{u, \underline{u}}$ of the covariant derivatives $D_{3}, D_{4}$. Observe that,

$$
\begin{aligned}
\omega & =-\frac{1}{2} \nabla_{4}(\log \Omega), & \underline{\omega} & =-\frac{1}{2} \nabla_{3}(\log \Omega), \\
\eta_{a} & =\zeta_{a}+\nabla_{a}(\log \Omega), & \underline{\eta}_{a} & =-\zeta_{a}+\nabla_{a}(\log \Omega) .
\end{aligned}
$$

We recall the integral formulas ${ }^{7}$ for a scalar function $f$ in $\mathcal{D}$,

$$
\begin{aligned}
& \frac{d}{d \underline{u}} \int_{S_{u, \underline{u}}} f=\int_{S_{u, \underline{u}}}\left(\frac{d f}{d \underline{u}}+\Omega \operatorname{tr} \chi f\right)=\int_{S_{u, \underline{u}}} \Omega\left(e_{4}(f)+\operatorname{tr} \chi f\right), \\
& \frac{d}{d u} \int_{S_{u, \underline{u}}} f=\int_{S_{u, \underline{u}}}\left(\frac{d f}{d u}+\Omega \operatorname{tr} \underline{\chi} f\right)=\int_{S_{u, \underline{u}}} \Omega\left(e_{3}(f)+\operatorname{tr} \underline{\chi} f\right) .
\end{aligned}
$$

In particular,

$$
\frac{d r}{d \underline{u}}=\frac{1}{8 \pi} \int_{S_{u, \underline{u}}} \Omega \operatorname{tr} \chi, \quad \frac{d r}{d u}=\frac{1}{8 \pi} \int_{S_{u, \underline{u}}} \Omega \operatorname{tr} \underline{\chi} .
$$

We also recall the following commutation formulae ${ }^{8}$ between $\nabla$ and $\nabla_{4}, \nabla_{3}$ in [4]:

Lemma 1. For a scalar function $f$ :

$$
\begin{aligned}
& {\left[\nabla_{4}, \nabla\right] f=\frac{1}{2}(\eta+\underline{\eta}) D_{4} f-\chi \cdot \nabla f} \\
& {\left[\nabla_{3}, \nabla\right] f=\frac{1}{2}(\eta+\underline{\eta}) D_{3} f-\underline{\chi} \cdot \nabla f .}
\end{aligned}
$$

\footnotetext{
${ }^{7}$ see for example Lemma 3.1.3 in [4].

${ }^{8}$ Here, $\epsilon_{a b}$ denotes the area 2 -form and div the divergence operator on the sphere $S_{u, \underline{u}}$. We refer the readers to [5] for the precise definitions of all of the notations below.
} 
For a 1-form tangent to $S$ :

$$
\begin{aligned}
{\left[\nabla_{4}, \nabla_{a}\right] U_{b} } & =-\chi_{a c} \nabla_{c} U_{b}+\epsilon_{a c} * \beta_{b} U_{c}+\frac{1}{2}\left(\eta_{a}+\underline{\eta}_{a}\right) D_{4} U_{b} \\
& -\chi_{a c} \underline{\eta}_{b} U_{c}+\chi_{a b} \underline{\eta} \cdot U \\
{\left[\nabla_{3}, \nabla_{a}\right] U_{b} } & =-\underline{\chi}_{a c} \nabla_{c} U_{b}+\epsilon_{a c}^{*} \underline{\beta}_{b} U_{c}+\frac{1}{2}\left(\eta_{a}+\underline{\eta}_{a}\right) D_{3} U_{b} \\
& -\underline{\chi}_{a c} \eta_{b} U_{c}+\underline{\chi}_{a b} \eta \cdot U .
\end{aligned}
$$

In particular,

$$
\begin{aligned}
& {\left[\nabla_{4}, \operatorname{div}\right] U=-\frac{1}{2} \operatorname{tr} \chi \operatorname{div} U-\hat{\chi} \cdot \nabla U-\beta \cdot U+\frac{1}{2}(\eta+\underline{\eta}) \cdot \nabla_{4} U-\underline{\eta} \cdot \hat{\chi} \cdot U,} \\
& {\left[\nabla_{3}, \operatorname{div}\right] U=-\frac{1}{2} \operatorname{tr} \underline{\chi} \operatorname{div} U-\underline{\hat{\chi}} \cdot \nabla U+\underline{\beta} \cdot U+\frac{1}{2}(\eta+\underline{\eta}) \cdot \nabla_{3} U-\eta \cdot \underline{\hat{\chi}} \cdot U .}
\end{aligned}
$$

1.2. Main theorem. For simplicity of our presentation ${ }^{9}$ we describe our main result in the context of the class of initial data used by Christodoulou. This class of initial data gives rise to a class of spacetimes endowed with a double null foliation as described in the previous subsection. As in [2], we prescribe the null incoming data to be trivial, i.e. corresponding to null cones in Minkowski space. In particular $S_{0,0}$ is the standard sphere of radius 1 . To prescribe null data on $H_{0}$ amounts to prescribe an arbitrary symmetric, traceless, smooth tensor $\hat{\chi}_{0}$, called initial shear. In [2], the initial shear is prescribed only in a region with a short characteristic length scale, i.e., $0 \leq \underline{u} \leq \delta$.

Definition 1. Given $\delta>0$ and $B>0$, we say that a smooth shear $\hat{\chi}_{0}$, supported on $H_{0}^{(0, \delta)}$, verifies Christodoulou's $\delta$-short pulse condition with constant $B$ if,

$$
\sup _{\underline{u}} \sum_{i \leq 5} \sum_{k \leq 3} \delta^{\frac{1}{2}+k}\left\|\nabla_{4}^{k} \nabla^{i} \hat{\chi}_{0}\right\|_{L^{\infty}\left(S_{0, \underline{u}}\right)} \leq B .
$$

It is proved in [2] that given any $B>0$ and $u_{*}<1$, there exists $\delta$ sufficiently small such that any prescribed shear $\chi_{0}$ satisfying (12) gives rise to a unique smooth spacetime $\mathcal{D}\left(u_{*}, \delta\right)$. In particular, the final inner sphere $S_{u_{*}, 0}$ has radius

$$
r_{*}=1-u_{*},
$$

which can be arbitrarily small, as long as $\delta$ is also chosen to be sufficiently small. This is summarized in the following theorem:

Theorem 1 (Christodoulou $\left.{ }^{10}[2]\right)$. For every $B>0$ and $u_{*}<1$ there exists $\delta>0$ sufficiently small such that if the null shear $\hat{\chi}_{0}$ along $H_{0}$ verifies Christodoulou's $\delta$-short pulse condition with constant $B$, then the future development of the corresponding initial data contains $\mathcal{D}\left(u_{*}, \delta\right)$ as a regular spacetime in which, in particular, the conditions MA1-MA4 below are satisfied, with $\delta_{0}=$ $\delta^{1 / 2}$.

\footnotetext{
${ }^{9}$ Similar results can be derived using the classes of initial data discussed in [5], [6] and [7].

${ }^{10}$ Strictly speaking, the original theorem of Christodoulou is stated somewhat differently. The version we use here is a straightforward reformulation of his results in [2] by a simple rescaling.
} 
MA1. $\Omega$ is comparable with its initial value

$$
\Omega=1+O\left(\delta_{0}\right) .
$$

MA2. The Ricci coefficients $\chi, \omega, \eta, \underline{\eta}, \nabla(\log \Omega), \underline{\chi}, \underline{\omega}$ verify

$$
\begin{aligned}
|\hat{\chi}, \omega| & =O\left(\delta^{-1 / 2}\right), \\
|\operatorname{tr} \chi| & =O(1), \\
\left|\eta, \underline{\eta}, \underline{\hat{\chi}}, \operatorname{tr} \underline{\chi}+\frac{2}{r}, \underline{\omega}, \nabla(\log \Omega)\right| & =O\left(\delta_{0}\right) .
\end{aligned}
$$

MA3. The derivatives of Ricci coefficients satisfy

$$
\begin{aligned}
|\nabla \eta| & =O\left(\delta_{0} \delta^{-1 / 2}\right), \\
|\nabla \underline{\hat{\chi}}, \nabla \operatorname{tr} \underline{\underline{\chi}}, \nabla \underline{\omega}| & =O\left(\delta_{0}\right) .
\end{aligned}
$$

MA4. $\operatorname{tr} \chi$ is close to its Minkowskian value on the initial cone $\underline{H}_{0}$

$$
\left|\operatorname{tr} \chi-\frac{2}{r}\right|=O\left(\delta_{0}\right), \quad \text { on } \underline{H}_{0} .
$$

To show that a trapped surface forms in $\mathcal{D}\left(u_{*}, \delta\right)$, Christodoulou needs in addition a uniform lower bound on the function $M_{0}=M_{0}\left[\hat{\chi}_{0}\right]$ defined on $S_{0,0}$ as follows,

$$
M_{0}(\omega)=M_{0}\left[\hat{\chi}_{0}\right](\omega):=\int_{0}^{\delta}\left|\hat{\chi}_{0}\right|^{2}\left(\underline{u}^{\prime}, \omega\right) d \underline{u}^{\prime},
$$

where the integral is taken along the null geodesic generators on $H_{0}$, transversal to $S_{0,0}$, initiating at $\omega \in S_{0,0}$. More precisely, he proved

Theorem 2 (Christodoulou [2]). Assume that the initial null shear $\hat{\chi}_{0}$ along $H_{0}$ verifies both Christodoulou's $\delta$-short pulse condition with constant $B$ and the isotropic condition

$$
\inf _{\omega \in S_{0,0}} M_{0}(\omega) \geq M_{*}>0 .
$$

Then, for any given $r_{*}>0$,

$$
r_{*}<\frac{M_{*}}{2}, \quad u_{*}=1-r_{*},
$$

there exists $\delta>0$ sufficiently small (depending only on $B$ and $M_{*}$ ) such that surface $S_{u_{*}, \delta} \subset \mathcal{D}\left(u_{*}, \delta\right.$ ), with $\mathcal{D}\left(u_{*}, \delta\right)$ constructed by Theorem 1, is necessarily trapped.

We now state our main result, which replaces the condition of the uniform lower bound (14) by merely the condition that $M_{0}$ is positive somewhere: 
Theorem 3 (Main theorem). Assume that the initial null shear $\hat{\chi}_{0}$ along $H_{0}$ verifies both Christodoulou's $\delta$-short pulse condition with constant $B$ and the following anisotropic condition

$$
\sup _{\omega \in S_{0,0}} M_{0}(\omega)>0 \text {. }
$$

Then, there exists $u_{*}>0$ sufficiently close to 1 and $\delta>0$ sufficiently small (depending on $B$ and the function $M_{0}$ ) such that the future development $\mathcal{D}\left(u_{*}, \delta\right)$, constructed by Theorem 1 , contains a trapped surface.

By continuity of $M_{0}$, the condition (15) implies that there exists $\epsilon$ and $M_{*}$ such that

$$
\inf _{\omega \in B_{p}(\epsilon)} M_{0}(\omega) \geq M_{*}>0
$$

where $B_{p}(\epsilon)$ is a geodesic ball of radius $\epsilon$ around some $p \in S_{0,0}$. Our main theorem therefore follows from the more quantitative version below:

Theorem 4. Assume that the initial null shear $\hat{\chi}_{0}$ along $H_{0}$ verifies both Christodoulou's $\delta$-short pulse condition with constant $B$ and the non-isotropic condition (16) with constants $\epsilon$ and $M_{*}$. Then, for any given $r_{*}>0$ verifying

$$
r_{*}=c_{0} M_{*} \epsilon^{5}, \quad u_{*}=1-r_{*},
$$

(where $c_{0}$ is a universal constant) there exists $\delta>0$ sufficiently small (depending on $B, M_{*}$ and $\epsilon$ ) such that the future development $\mathcal{D}\left(u_{*}, \delta\right)$, constructed by Theorem 1, contains a trapped surface of area at least $\gtrsim M_{*}^{2} \epsilon^{10}$.

Remark 1. In Chapter 2 of [2], Christodoulou also constructed a class of initial data satisfying the assumptions of Theorems 1 and 2. The construction, which is based on solving ordinary differential equations along the null generators of the initial outgoing null hypersurface, can also be applied to construct initial data which obey the assumptions of our main theorem (Theorem 3). In particular, since the initial data at any point $p$ on the initial hypersurface depend only on the prescribed conformal part of the metric along the null generator passing through $p$, we can prescribe initial data which are flat except in a small neighborhood of an outgoing generator on the initial hypersurface.

Remark 2. The conditions MA1-MA4 are needed to implement part (2) of our main theorem. Though strictly speaking Christodoulou's theorem implies MA1-MA4 with $\delta_{0}=\delta^{1 / 2}$, we prefer to write them in this more general form with respect to a second parameter $\delta_{0}$, such that $\delta_{0}, \delta_{0}^{-1} \delta$ are sufficiently small. This formulation allows us to adapt our result to the more general initial data used in [5], [6] and [7].

Remark 3. The original theorem of Christodoulou [2] also applies to the case when the initial arearadius is $r_{0}$. In that case, (12) can be replaced by $\sup _{\underline{u}} \sum_{i \leq 5} \sum_{k \leq 3} \delta^{\frac{1}{2}+k} r_{0}^{1+i}\left\|\nabla_{4}^{k} \nabla^{i} \hat{\chi}_{0}\right\|_{L^{\infty}\left(S_{0, \underline{u}}\right)} \leq B$, and the definition of $M$ is similarly modified, i.e. $M\left(\omega ; r_{0}\right)=\int_{0}^{\delta} r_{0}^{2}\left|\hat{\chi}_{0}\right|^{2}\left(\underline{u}^{\prime}, \omega\right) d \underline{u}^{\prime}$. With this definition, if the initial data satisfy $\inf _{\omega \in S_{0,0}} M_{0}\left(\omega ; r_{0}\right) \geq M_{*}>0$, the formation of trapped surface theorem (Theorem 2) still holds after choosing $\delta$ sufficiently small depending on $B$ and $M_{*}$. By the same rescaling argument our main theorem (Theorem 3) holds in the case where the initial area-radius is 
$r_{0}$ if the condition $\sup _{\omega \in S_{0,0}} M_{0}\left(\omega ; r_{0}\right)>0$ is verified. Moreover, in [2], Christodoulou proved that one can take $r_{0} \rightarrow \infty$ and construct a spacetime from null infinity. For such spacetimes, our anisotropic condition can be replaced by the condition $\sup _{\omega \in S_{0,0}} M_{0}(\omega ; \infty)>0$ and still guarantee the formation of a trapped surface.

Remark 4. Note that while in [2] the desired trapped surface can be found among the surfaces $S_{u, u}$, consistent with the double null foliation, this is no longer the case in our theorem. Instead, we will identify a different 2-sphere, embedded in the null hypersurface $\{\underline{u}=\delta\}$, which can be shown to be trapped. Note also that the function $M_{0}[\hat{\chi}](\omega)=\int_{0}^{\delta}|\hat{\chi}(\underline{u}, \omega)|^{2} d \underline{u}^{\prime}$ is invariant ${ }^{11}$ with respect to a change of foliation along the initial hypersurface $H_{0}$. It is thus impossible to change the foliation on the initial hypersurface $H_{0}$ so that the isotropic condition in Theorem 2 holds.

We show that a trapped surface exists in $\mathcal{D}\left(u_{*}, \delta\right)$ by finding an embedded trapped 2-sphere in the incoming null hypersurface $\underline{H}_{\delta}$. Notice that by MA2, $\operatorname{tr} \chi<0$ on $\underline{H}_{\delta}$. Therefore, it suffices to find a 2 -sphere such that the outgoing null expansion is also pointwise negative. We will achieve this in two steps. In Section 2 we make use of the conditions MA1-MA4 to reduce the problem of existence of a trapped surface to that of finding appropriate solutions to an elliptic inequality, see (20), on $S_{0,0}$. In Section 3, we prove that under the assumption (16) of the main theorem, a desired solution to the elliptic inequality exists.

\section{Reduction to AN ELliptic INEQUALity on the initial SPHERE $S_{0,0}$}

In this section, we show that under the assumptions MA1-MA4, the existence of a trapped surface can be reduced to constructing a solution to an elliptic inequality (20). The main result of the section is stated in Theorem 5 .

We first make a remark concerning the global parametrization of points in $\mathcal{D}\left(u_{*}, \delta\right)$ by $u, \underline{u}$ and coordinates $\omega$ on $S_{0,0}$.

Proceeding as in [2] we associate to each coordinate patch on $S_{0,0}$, a system of transported coordinates defined by

$$
\mathcal{A}_{\Omega e_{4}} \theta^{a}=0, \quad \text { on } H_{0}
$$

and

$$
\mathcal{L}_{\Omega e_{3}} \theta^{a}=0, \quad \text { in } \mathcal{D}\left(u_{*}, \delta\right),
$$

where $\mathbb{A}$ is the restriction of the Lie derivative to $T S_{u, \underline{u}}$ (see [2], chapter 1). This provides an identification of each point in the spacetime $\mathcal{D}\left(u_{*}, \delta\right)$ with a point in the initial sphere $S_{0,0}$ by the value of the coordinate functions.

\footnotetext{
${ }^{11}$ In other words $M_{0}[\hat{\chi}](\omega)=M_{0}\left[\hat{\chi}^{\prime}\right](\omega)=\int_{f(0, \omega)}^{f(\delta, \omega)}\left|\hat{\chi}^{\prime}\left(\underline{u}^{\prime}, \omega\right)\right|^{2} d \underline{u}^{\prime}$ where $\hat{\chi}_{a b}^{\prime}=\frac{1}{2} g\left(D_{e_{a}} \frac{\partial}{\partial \underline{u}^{\prime}}, e_{b}\right)$ for a new foliation $\underline{u}^{\prime}=f(\underline{u}, \omega)$, with $\frac{\partial f}{\partial \underline{u}}>0$.
} 
It follows that any point in $\mathcal{D}\left(u_{*}, \delta\right)$ can also be uniquely specified by the coordinates $(\underline{u}, u, \omega)$, where $\omega \in S_{0,0}$.

We can now state the main result of this section:

Theorem 5. Assume that the spacetime $\mathcal{D}\left(u_{*}, \delta\right)$ satisfies MA1-MA4. Let $M_{0}$ be the function on the initial sphere $S_{0,0}$ defined by (13), i.e.,

$$
M_{0}(\omega):=\int_{0}^{\delta}|\hat{\chi}|^{2}\left(u=0, \underline{u}^{\prime}, \omega\right) d \underline{u}^{\prime} .
$$

Assume $R$ is a smooth function on $S_{0,0}$ satisfying $r_{*}+C \delta_{0}<R<1$ as well as the elliptic inequality ${ }^{12}$ on $S_{0,0}$

$$
-\Delta_{0} R+R^{-1}\left|\nabla_{0} R\right|^{2}+R<2^{-1} M_{0}-C \delta_{0},
$$

with $C>0$ a constant depending only on $\sum_{i \leq 2}\left\|\nabla_{0}^{i} R\right\|_{L^{\infty}\left(S_{0,0}\right)}$.

Then, for $\delta \delta_{0}^{-1}, \delta_{0}$ sufficiently small, the 2-sphere defined by $\{(\underline{u}, u, \omega): \underline{u}=\delta, 1-u=R(\omega)\}$ is a trapped surface.

We prove Theorem 5 in this section and leave for the next section the task to show that a smooth solution $R$ to (20) exists, provided that $M_{0}$ satisfies the assumptions of our main theorem.

The proof of Theorem 5 will be achieved in two steps. In Section 2.1, we will carry out Christodoulou's argument in [2] to estimate $\operatorname{tr} \chi$ with respect to the original foliation on $\underline{H}_{\delta}$. In Section 2.2 , we will then deform the foliation on $\underline{H}_{\delta}$ in such a way that the 2-sphere $\{(\underline{u}, u, \omega): \underline{u}=\delta, 1-u=R(\omega)\}$ is a level surface adapted to the new foliation. We then compute the desired outgoing expansion $\operatorname{tr} \chi^{\prime}$ on the 2-sphere $\{(\underline{u}, u, \omega): \underline{u}=\delta, 1-u=R(\omega)\}$.

We note that the derivation in Section 2.2 can be simplified by taking into account the specific properties of the spacetime constructed in Theorem 1. We will include the simplified argument in Section 2.3. Nevertheless, we prefer to proceed below with the general derivation in view of possible applications to a more general setting.

2.1. Christodoulou's argument. In [2], it was shown that under the assumptions MA1-MA4, the expansion $\operatorname{tr} \chi$ on each of the spheres $S_{u, \delta}$ on $\underline{H}_{\delta}$ can be computed up to a small error depending on $\delta$. In the context of [2], where a uniform lower bound on $M_{0}$ is assumed, this is sufficient to conclude the existence of a trapped surface $S$ of the form $S=S_{u, \delta}$. In the case of our weaker condition (16), his argument only shows that $\operatorname{tr} \chi$ becomes sufficiently negative in part of the sphere $S_{u, \delta}$. To obtain a trapped surface we need to combine that fact with a new deformation argument of the foliation on $\underline{H}_{\delta}$.

\footnotetext{
${ }^{12}$ Here $\Delta_{0}$ and $\nabla_{0}$ are defined with respect to the connection on the initial sphere $S_{0,0}$.
} 
Christodoulou's argument for the formation of trapped surfaces in [2] rests on the equations ${ }^{13}$,

$$
\begin{aligned}
\nabla_{4} \operatorname{tr} \chi & =-|\hat{\chi}|^{2}-\frac{1}{2}(\operatorname{tr} \chi)^{2}-2 \omega \operatorname{tr} \chi \\
\nabla_{3} \hat{\chi}+\frac{1}{2} \operatorname{tr} \underline{\chi} \hat{\chi} & =\nabla \widehat{\otimes} \eta+2 \underline{\omega} \hat{\chi}-\frac{1}{2} \operatorname{tr} \chi \underline{\hat{\chi}}+\eta \hat{\otimes} \eta
\end{aligned}
$$

In view of our Ricci coefficients assumptions we can rewrite,

$$
\begin{aligned}
\nabla_{4} \operatorname{tr} \chi & =-|\hat{\chi}|^{2}+O\left(\delta^{-1 / 2}\right) \\
\nabla_{3} \hat{\chi}+\frac{1}{2} \operatorname{tr} \underline{\chi} \hat{\chi} & =O\left(\delta_{0} \delta^{-1 / 2}\right)
\end{aligned}
$$

Multiplying the second equation by $\hat{\chi}$,

$$
\nabla_{3}|\hat{\chi}|^{2}+\operatorname{tr} \underline{\chi}|\hat{\chi}|^{2}=O\left(\delta_{0} \delta^{-1}\right)
$$

Using also our assumptions for $u, \underline{u}, \Omega$ we deduce ${ }^{14}$,

$$
\begin{aligned}
\frac{\partial}{\partial \underline{u}} \operatorname{tr} \chi & =-|\hat{\chi}|^{2}+O\left(\delta^{-1 / 2}\right) \\
\frac{\partial}{\partial u}|\hat{\chi}|^{2}+\operatorname{tr} \underline{\chi}|\hat{\chi}|^{2} & =O\left(\delta_{0} \delta^{-1}\right)
\end{aligned}
$$

Integrating (21) we obtain,

$$
\operatorname{tr} \chi(u, \underline{u})=\frac{2}{r(u, 0)}-\int_{0}^{\underline{u}}|\hat{\chi}|\left(u, \underline{u}^{\prime}\right)^{2} d \underline{u}^{\prime}+O\left(\delta_{0}\right)
$$

In view of our assumptions for tr $\underline{\chi}$ and $\frac{d r}{d u}$, (22) implies

$$
\begin{aligned}
\frac{d}{d u}\left(r^{2}|\hat{\chi}|^{2}\right) & =r^{2} \frac{d}{d u}|\hat{\chi}|^{2}+2 r \frac{d r}{d u}|\hat{\chi}|^{2}=r^{2}\left[-\operatorname{tr} \underline{\chi}|\hat{\chi}|^{2}+O\left(\delta_{0} \delta^{-1}\right)\right]+2 r\left[-1+O\left(r \delta_{0}\right)\right]|\hat{\chi}|^{2} \\
& =O\left(\delta_{0} \delta^{-1}\right) .
\end{aligned}
$$

Therefore,

$$
r^{2}|\hat{\chi}|^{2}(u, \underline{u})=r^{2}(0, \underline{u})|\hat{\chi}|^{2}(0, \underline{u})+O\left(\delta_{0} \delta^{-1}\right)
$$

Let $\hat{\chi}_{0}$ denote the initial data for $\hat{\chi}$ :

$$
\hat{\chi}_{0}(\underline{u})=\hat{\chi}(0, \underline{u}) .
$$

\footnotetext{
${ }^{13}$ The operator $\widehat{\otimes}$ referes to the traceless part of the symmetrized tensor product. We refer the readers to [5] for the precise notations used in this paper.

${ }^{14}$ Here, $\frac{\partial}{\partial \underline{u}}$ and $\frac{\partial}{\partial u}$ refers to the coordinate vector fields defined with respect to a coordinate system $\left(u, \underline{u}, \theta^{1}, \theta^{2}\right)$, where $\theta^{1}$ and $\theta^{2}$ satisfy the conditions (18) and (19). Notice that this is different from the vector fields $\Omega e_{4}$ and $\Omega e_{3}$ as $\Omega e_{4}$ would have an "angular component" in the coordinate system $\left(u, \underline{u}, \theta^{1}, \theta^{2}\right)$. In particular, $\frac{\partial}{\partial \underline{u}}$ is not parallel to the null generators. Nevertheless, as a consequence of the assumptions MA1-MA4, the difference between $\frac{\partial}{\partial \underline{u}}$ and $\Omega e_{4}$ is a vector field on $T S_{u, \underline{u}}$ with a small norm and will be collected in the error terms in the equations.
} 
We deduce,

$$
|\hat{\chi}|^{2}(u, \underline{u})=\frac{r^{2}(0, \underline{u})}{r^{2}(u, \underline{u})}\left|\hat{\chi}_{0}\right|^{2}(\underline{u})+O\left(\delta_{0} \delta^{-1}\right)
$$

Since $r(u, \underline{u})=r(u, 0)+O(\delta)$,

$$
|\hat{\chi}|^{2}(u, \underline{u})=\frac{1}{r^{2}(u, 0)}\left|\hat{\chi}_{0}\right|^{2}(\underline{u})+O\left(\delta_{0} \delta^{-1}\right) .
$$

Thus, returning to (23), and recalling that

$$
M_{0}(\omega)=\int_{0}^{\delta}\left|\hat{\chi}_{0}\right|^{2}\left(\underline{u}^{\prime}, \omega\right) d \underline{u}^{\prime},
$$

we deduce the following:

Proposition 1. Under the assumptions MA1-MA4 we have, for $\delta_{0}, \delta_{0}^{-1} \delta$ sufficiently small,

$$
\operatorname{tr} \chi(u, \underline{u}=\delta, \omega)=\frac{2}{r(u, 0)}-\frac{1}{r^{2}(u, 0)} M_{0}(\omega)+O\left(\delta_{0}\right)
$$

Since $r(u, 0)=1-u+O\left(\delta_{0}\right)$, this implies

Corollary 1. For $\delta_{0}, \delta_{0}^{-1} \delta$ small, the necessary and sufficient condition to have tr $\chi<0$ everywhere on the sphere $S_{u, \delta}$ is that

$$
2(1-u)<M_{0}(\omega)-O\left(\delta_{0}\right)
$$

holds uniformly for every $\omega \in S_{0,0}$.

Under the assumptions of our main theorem, we can only hope that the outgoing null expension tr $\chi$ adapted to the foliation $(u, \underline{u})$ becomes negative in the part where $M_{0}$ is positive. Thus to prove our main theorem, we need to combine this argument with the new deformation mechanism which leads to the formation of a trapped surface that is no longer adapted to the double null foliation $(u, \underline{u})$. Instead, as stated in Theorem 5, the trapped surface will be a topological 2-sphere embedded in the incoming null hypersurface $\{\underline{u}=\delta\}$ defined by $\{(\underline{u}, u, \omega): \underline{u}=\delta, 1-u=R(\omega)\}$.

2.2. Main transformation formula. According to the statement of Theorem $5,\{(\underline{u}, u, \omega): \underline{u}=$ $\delta, 1-u=R(\omega)\}$ will correspond to a trapped surface provided that $R$ satisfies (20). To verify that, we need to compute its null expansion, which differs from the null expansion tr $\chi$ relative to the double null foliation $(u, \underline{u})$ restricted to $\{(\underline{u}, u, \omega): \underline{u}=\delta, 1-u=R(\omega)\}$. To compute the correct null expansion $\operatorname{tr} \chi^{\prime}$, we introduce the new null frame adapted to this set,

$$
e_{3}^{\prime}=e_{3}, \quad e_{a}^{\prime}=e_{a}-\Omega e_{a}(R) e_{3}, \quad e_{4}^{\prime}=e_{4}-2 \Omega e^{a}(R) e_{a}+\Omega^{2}|\nabla R|^{2} e_{3}
$$

Recall that by definition $e_{3}(u)=\Omega^{-1}$. Thus we have, $e_{a}^{\prime}(u+R-1)=e_{a}(R)-e_{a}(R) \Omega e_{3}(u)=0$. Also, since $e_{3}$ is orthogonal to any vector tangent to $\underline{H}$ we easily check that

$$
g\left(e_{a}^{\prime}, e_{b}^{\prime}\right)=g\left(e_{a}, e_{b}\right)=\delta_{a b}, \quad g\left(e_{4}^{\prime}, e_{a}^{\prime}\right)=g\left(e_{4}^{\prime}, e_{4}^{\prime}\right)=0, \quad g\left(e_{3}^{\prime}, e_{4}^{\prime}\right)=-2 .
$$


We prove the following

Proposition 2. The trace of the null second fundamental form $\chi^{\prime}$, relative to the new frame (27), is given by

$$
\operatorname{tr} \chi^{\prime}=\operatorname{tr} \chi-2 \Omega \Delta R-4 \Omega \eta \cdot \nabla R-4 \Omega^{2} \underline{\hat{\chi}}_{b c} \nabla^{b} R \nabla^{c} R-\Omega^{2} \operatorname{tr} \underline{\underline{\chi}}|\nabla R|^{2}-8 \Omega^{2} \underline{\omega}|\nabla R|^{2} .
$$

Proof. Let $F_{a}=\Omega\left(\nabla_{a} R\right)$. We can then write $e_{4}^{\prime}=e_{4}-2 F+|F|^{2} e_{3}$ with $F=F^{c} e_{c}$ and $e_{b}^{\prime}=e_{b}-F_{b} e_{3}$. We have,

$$
\chi^{\prime}\left(e_{a}^{\prime}, e_{b}^{\prime}\right):=g\left(D_{a^{\prime}} e_{4}^{\prime}, e_{b}^{\prime}\right)=g\left(D_{a} e_{4}^{\prime}, e_{b}^{\prime}\right)-F_{a} g\left(D_{3} e_{4}^{\prime}, e_{b}^{\prime}\right)
$$

The first term is given by

$$
\begin{aligned}
g\left(D_{a} e_{4}^{\prime}, e_{b}^{\prime}\right) & =g\left(D_{a}\left(e_{4}-2 F+|F|^{2} e_{3}\right), e_{b}-F_{b} e_{3}\right) \\
& =\chi\left(e_{a}, e_{b}\right)-2 F_{b} \zeta_{a}-2 \nabla_{a} F_{b}+2 F_{b} g\left(D_{a} F, e_{3}\right)+|F|^{2} g\left(D_{a} e_{3}, e_{b}-F_{b} e_{3}\right) \\
& =\chi_{a b}-2 \zeta_{a} F_{b}-2 \nabla_{a} F_{b}-2 F_{b} \underline{\chi}\left(F, e_{a}\right)+|F|^{2} \underline{\chi}_{a b} \\
& =\chi_{a b}-2 \zeta_{a} F_{b}-2 \nabla_{a} F_{b}-2 F_{b} F^{c} \underline{\chi}_{a c}+|F|^{2} \underline{\chi}_{a b}
\end{aligned}
$$

Also,

$$
\begin{aligned}
g\left(D_{3} e_{4}^{\prime}, e_{b}^{\prime}\right) & =g\left(D_{3}\left(e_{4}-2 F+|F|^{2} e_{3}\right), e_{b}-F_{b} e_{3}\right) \\
& =g\left(D_{3} e_{4}, e_{b}\right)-F_{b} g\left(D_{3} e_{4}, e_{3}\right)-2 \nabla_{3} F_{b} \\
& =2 \eta_{b}+4 F_{b} \underline{\omega}-2 \nabla_{3} F_{b}
\end{aligned}
$$

Hence,

$$
\begin{aligned}
\chi_{a b}^{\prime} & =\chi_{a b}-2 \zeta_{b} F_{a}-2 \nabla_{a} F_{b}-2 F_{b} F^{c} \underline{\chi}_{a c}+|F|^{2} \underline{\chi}_{a b}-F_{a}\left(2 \eta_{b}+4 F_{b} \underline{\omega}-2 \nabla_{3} F_{b}\right) \\
& =\chi_{a b}-2 \nabla_{a} F_{b}+2 F_{a} \nabla_{3} F_{b}-2 \zeta_{b} F_{a}-2 F_{a} \eta_{b}+|F|^{2} \underline{\chi}_{a b}-2 F_{b} F^{c} \underline{\chi}_{a c}-4 \underline{\omega} F_{a} F_{b}
\end{aligned}
$$

By symmetry in $a, b$ we deduce the formula,

$$
\begin{aligned}
\chi_{a b}^{\prime}=\chi_{a b} & -\left(\nabla_{a} F_{b}+\nabla_{b} F_{a}\right)+\nabla_{3}\left(F_{a} F_{b}\right)-\left(\zeta_{b}+\eta_{b}\right) F_{a}-\left(\zeta_{a}+\eta_{a}\right) F_{b} \\
& +|F|^{2} \underline{\chi}_{a b}-F_{b} F^{c} \underline{\chi}_{a c}-F_{a} F^{c} \underline{\chi}_{b c}-4 \underline{\omega} F_{a} F_{b}
\end{aligned}
$$

and, taking the trace,

$$
\begin{aligned}
\operatorname{tr} \chi^{\prime} & =\operatorname{tr} \chi-2 \operatorname{div} F+\nabla_{3}|F|^{2}-2(\eta+\zeta) \cdot F+\left(|F|^{2} \operatorname{tr} \underline{\chi}-2 \underline{\chi}_{b c} F^{b} F^{c}\right)-4 \underline{\omega}|F|^{2} \\
& =\operatorname{tr} \chi-2 \operatorname{div} F+\nabla_{3}|F|^{2}-2(\eta+\zeta) \cdot F-2 \underline{\hat{\chi}} b c F^{b} F^{c}-4 \underline{\omega}|F|^{2} .
\end{aligned}
$$

We next calculate $\nabla_{3}|F|^{2}$ using

$$
\nabla_{3} R=0
$$

and the commutation formula

$$
\left[\nabla_{3}, \nabla\right] h=(\nabla \log \Omega) \nabla_{3} h-\underline{\chi} \cdot \nabla h .
$$


Since $F=\Omega \nabla R$ we deduce,

$$
\begin{aligned}
\nabla_{3} F_{a} & =\nabla_{3}(\Omega \nabla R)=\Omega\left(\nabla_{3} \nabla R\right)+\left(\nabla_{3} \Omega\right) \nabla R \\
& =\Omega \nabla \nabla_{3} R+(\nabla \log \Omega) \Omega \nabla_{3} R-\Omega \underline{\chi} \cdot \nabla R-2 \underline{\omega} \Omega \nabla R \\
& =-\Omega \underline{\chi} \cdot \nabla R-2 \underline{\omega} \Omega \nabla R .
\end{aligned}
$$

from which we derive,

$$
\nabla_{3}|F|^{2}=-\Omega^{2} \operatorname{tr} \underline{\chi}|\nabla R|^{2}-2 \Omega^{2} \underline{\hat{\chi}}_{b c} \nabla_{b} R \nabla_{c} R-4 \Omega^{2} \underline{\omega}|\nabla R|^{2} .
$$

Therefore,

$$
\begin{aligned}
\operatorname{tr} \chi^{\prime}= & \operatorname{tr} \chi-2 \operatorname{div} F-2(\eta+\zeta) \cdot F-2 \underline{\hat{\chi}}_{b c} F^{b} F^{c}-4 \underline{\omega}|F|^{2} \\
& -\Omega^{2} \operatorname{tr} \underline{\chi}|\nabla R|^{2}-2 \Omega^{2} \underline{\hat{\chi}}_{b c} \nabla_{b} R \nabla_{c} R-4 \Omega^{2} \underline{\omega}|\nabla R|^{2} \\
= & \operatorname{tr} \chi-2 \Omega \Delta R-4 \Omega \eta \cdot \nabla R-4 \Omega^{2} \underline{\hat{\chi}}_{b c} \nabla_{b} R \nabla_{c} R-\Omega^{2} \operatorname{tr} \underline{\chi}|\nabla R|^{2}-8 \Omega^{2} \underline{\omega}|\nabla R|^{2}
\end{aligned}
$$

as desired.

We combine this with the main assumptions MA1-MA4 to derive the following proposition:

Proposition 3. The trace of the null second fundamental form $\chi^{\prime}$, relative to the new frame (27), evaluated at the set $\{(\underline{u}, u, \omega): \underline{u}=\delta, 1-u=R(\omega)\}$ is given by

$$
\operatorname{tr} \chi^{\prime}(u=1-R(\omega), \underline{u}=\delta, \omega)=\operatorname{tr} \chi(u=1-R(\omega), \underline{u}=\delta, \omega)-\frac{2 \Delta_{0} R}{R^{2}}+\frac{2\left|\nabla_{0} R\right|^{2}}{R^{3}}+O_{R}\left(\delta_{0}\right),(30
$$

where $O_{R}\left(\delta_{0}\right)$ denotes a term bounded by $C \delta_{0}$, where $C$ depends only on the $L^{\infty}$ norm of $R, \nabla_{0} R$ and $\nabla_{0}^{2} R$, and $\nabla_{0}, \Delta_{0}$, as before, are defined with respect to the connection on the initial sphere $S_{0,0}$.

Proof. By MA1-MA4, $\Omega-1, \eta, \underline{\hat{\chi}}, \operatorname{tr} \underline{\chi}+\frac{2}{r}$ and $\underline{\omega}$ are small in terms of $\delta_{0}$. Thus

$$
\operatorname{tr} \chi^{\prime}(u=1-R(\omega), \underline{u}=\delta, \omega)=\operatorname{tr} \chi(u=1-R(\omega), \underline{u}=\delta, \omega)-2 \Delta R+\frac{2|\nabla R|^{2}}{R}+O_{R}\left(\delta_{0}\right) .
$$

To achieve the proposition, we need to compare ${ }^{15} \nabla R$ with $\nabla_{0} R$. To this end, we consider the equation

$$
\nabla_{3} R=0
$$

and commute with angular derivatives. The commutation formulae in Lemma 1 and MA1-MA4 imply that

$$
\nabla_{3} \nabla R+\frac{1}{2} \operatorname{tr} \underline{\chi} \nabla R=O_{R}\left(\delta_{0}\right)
$$

\footnotetext{
${ }^{15}$ Recall that $\nabla R$ is defined with respect to the connection coefficients of the spheres adapted to the $(u, \underline{u})$ foliation at the set $\{(\underline{u}, u, \omega): \underline{u}=\delta, 1-u=R(\omega)\}$; while $\nabla_{0} R$ is defined with respect to the connection coefficients on the initial sphere $S_{0,0}$.
} 
and

$$
\nabla_{3} \nabla^{2} R+\operatorname{tr} \underline{\chi} \nabla^{2} R=O_{R}\left(\delta_{0}\right) .
$$

This implies, via the condition $\left|\operatorname{tr} \underline{\chi}+\frac{2}{r}\right|=O\left(\delta_{0}\right)$ in MA2, that

$$
\nabla_{3}(r \nabla R)=O_{R}\left(\delta_{0}\right)
$$

and

$$
\nabla_{3}\left(r^{2} \nabla^{2} R\right)=O_{R}\left(\delta_{0}\right)
$$

Therefore,

$$
|\nabla R|^{2}=\frac{\left|\nabla_{0} R\right|^{2}}{R^{2}}+O_{R}\left(\delta_{0}\right), \quad \Delta R=\frac{\Delta_{0} R}{R^{2}}+O_{R}\left(\delta_{0}\right) .
$$

The conclusion thus follows.

We now combine the results of Propositions 1 and 3. According to Proposition 1, tr $\chi$ evaluated at $\{(\underline{u}, u, \omega): \underline{u}=\delta, 1-u=R(\omega)\}$ is given by

$$
\operatorname{tr} \chi(u=1-R(\omega), \underline{u}=\delta, \omega)=\frac{2}{R(u, 0)}-\frac{1}{R^{2}(u, 0)} \int_{0}^{\delta}\left|\hat{\chi}_{0}\right|^{2}\left(\underline{u}^{\prime}, \omega\right) d \underline{u}^{\prime}+O\left(\delta_{0}\right) .
$$

Thus, inserting in (30), we have

$$
\operatorname{tr} \chi^{\prime}(u=1-R(\omega), \underline{u}=\delta, \omega) \leq \frac{2}{R}-\frac{2 \Delta_{0} R}{R^{2}}+\frac{2\left|\nabla_{0} R\right|^{2}}{R^{3}}-\frac{1}{R^{2}} M_{0}-O_{R}\left(\delta_{0}\right) .
$$

where

$$
M_{0}=\int_{0}^{\delta}\left|\hat{\chi}_{0}\right|^{2}\left(\underline{u}^{\prime}\right) d \underline{u}^{\prime}
$$

This concludes the proof of Theorem 5 .

2.3. An alternative derivation. In the context of our specific situation we outline ${ }^{16}$ here a slightly easier argument leading to the formula for $\operatorname{tr} \chi^{\prime}$ on $\underline{H}_{\delta}$ in terms of $\operatorname{tr} \chi$ on $\underline{H}_{\delta}$ and the function $R$ in the limit $\delta \rightarrow 0$. Note however that the derivation below depends on further properties of the Ricci coefficients, in addition to MA1-MA4, proved in [2]. This provides an alternative proof of Theorem 5.

We recall from [2] that in the limit $\delta \rightarrow 0$, the following curvature components tend to zero:

$$
\underline{\beta} \rightarrow 0, \quad \underline{\alpha} \rightarrow 0
$$

Also,

$$
\underline{\hat{\chi}}=O\left(\delta^{\frac{1}{2}}\right), \quad \operatorname{tr} \underline{\chi} \rightarrow-\frac{2}{r}
$$

\footnotetext{
${ }^{16}$ We thank Demetrios Christodoulou for pointing out a related idea.
} 
$\hat{\chi}$ is of the order $O\left(\delta^{-\frac{1}{2}}\right)$ and all the remaining Ricci coefficients are at least $O(1)$.

By (32), in the limit $\delta \rightarrow 0$

$$
\mathcal{L}_{\Omega e_{3}} \gamma=2 \underline{\chi}=-\frac{2}{r} \gamma
$$

holds along the incoming null hypersurface $\underline{H}_{\delta}$, where $\gamma$ is a induce Riemannian metric on $S_{u, \delta}$.

It follows that at the $\delta \rightarrow 0$ limit, the metric $\gamma^{\prime}$ on the sphere defined by $\{(\underline{u}, u, \omega): \underline{u}=\delta, 1-u=$ $R(\omega)\}$ is conformal to the metric on the standard sphere $\gamma_{0}$ with the conformal factor given by

$$
\gamma^{\prime}=R^{2} \gamma_{0}
$$

Let $K^{\prime}$ denote the Gauss curvature of the sphere $\{(\underline{u}, u, \omega): \underline{u}=\delta, 1-u=R(\omega)\}$ and $K_{0}$ be the Gauss curvature on the initial sphere $S_{0,0}$. Then, in view of (33),

$$
K^{\prime}=R^{-2}\left(K_{0}-\Delta_{0}(\log R)\right)=R^{-2}\left(1-\Delta_{0}(\log R)\right) .
$$

Consider the Gauss equation for the sphere $\{(\underline{u}, u, \omega): \underline{u}=\delta, 1-u=R(\omega)\}$,

$$
K^{\prime}=-\rho^{\prime}+\frac{1}{2} \hat{\chi}^{\prime} \cdot \underline{\hat{\chi}}^{\prime}-\frac{1}{4} \operatorname{tr} \chi^{\prime} \operatorname{tr} \underline{\chi^{\prime}} .
$$

Also the Gauss equation for the 2-spheres adapted to the original foliation,

$$
K=-\rho+\frac{1}{2} \hat{\chi} \cdot \underline{\hat{\chi}}-\frac{1}{4} \operatorname{tr} \chi \operatorname{tr} \underline{\chi} .
$$

We will derive $\operatorname{tr} \chi^{\prime}$ in terms of $\operatorname{tr} \chi$ and $R$ using equations (35) and (36). By definition, $e_{a}^{\prime}=$ $e_{a}-\Omega e_{a}(R) e_{3}$ and $e_{3}^{\prime}=e_{3}$. Since $e_{3}$ is normal to $e_{a}, e_{a}^{\prime}$ and $e_{3}$, and

$$
D_{e_{3}} e_{3}=-2 \omega e_{3},
$$

we have

$$
\underline{\chi}_{a b}^{\prime}=\frac{1}{2} g\left(D_{a^{\prime}} e_{3}, e_{b}^{\prime}\right)=\frac{1}{2} g\left(D_{a} e_{3}, e_{b}\right)=\underline{\chi}_{a b} .
$$

Therefore, as $\delta \rightarrow 0$,

$$
\operatorname{tr} \underline{\chi}^{\prime}=\operatorname{tr} \underline{\chi} \rightarrow-\frac{2}{r}
$$

and

$$
\left|\underline{\hat{\chi}}^{\prime}\right|=O\left(\delta^{\frac{1}{2}}\right) .
$$

On the other hand, $e_{4}^{\prime}=e_{4}-2 \Omega e_{a}(R) e_{a}+\Omega^{2}|\nabla R|^{2} e_{3}, \hat{\chi}^{\prime}$ can be expressed as $\hat{\chi}$ plus a linear combination of the Ricci coefficients with respect to the original frame which are bounded independent of $\delta$. Therefore,

$$
\left|\hat{\chi}^{\prime}-\hat{\chi}\right|=O(1)
$$


where the implicit constant may depend on $R$. Note that (37) and (38) together imply in the limit $\delta \rightarrow 0$,

$$
\left|\hat{\chi} \cdot \underline{\hat{\chi}}-\hat{\chi}^{\prime} \cdot \underline{\hat{\chi}}^{\prime}\right| \rightarrow 0
$$

Since $e_{4}^{\prime}=e_{4}-2 \Omega e_{a}(R) e_{a}+\Omega^{2}|\nabla R|^{2} e_{3}$ and $e_{3}^{\prime}=e_{3}, \rho^{\prime}-\rho$ can be written as a linear combination of $\underline{\beta}$ and $\underline{\alpha}$. Thus, by (31), in the limit $\delta \rightarrow 0$,

$$
\rho^{\prime} \rightarrow \rho .
$$

In the $\delta \rightarrow 0$ limit the Gauss equations (36) becomes

$$
\rho=-K-\frac{1}{4} \operatorname{tr} \chi \operatorname{tr} \underline{\chi}=-R^{-2}+\frac{1}{2 R} \operatorname{tr} \chi
$$

On the other hand, the $\delta \rightarrow 0$ limit of the Gauss equations (35) becomes

$$
K^{\prime}=-\rho^{\prime}-\frac{1}{4} \operatorname{tr} \chi^{\prime} \operatorname{tr} \underline{\chi}^{\prime}=-\rho+\frac{1}{2 R} \operatorname{tr} \chi^{\prime}=R^{-2}-\frac{1}{2 R} \operatorname{tr} \chi+\frac{1}{2 R} \operatorname{tr} \chi^{\prime}
$$

Thus, making use of (34), we have

$$
\begin{aligned}
\operatorname{tr} \chi^{\prime} & =\operatorname{tr} \chi-\frac{1}{2 R}+2 R K^{\prime}=\operatorname{tr} \chi-\frac{2}{R} \Delta_{0}(\log R) \\
& =\operatorname{tr} \chi+\frac{2}{R}\left(-\frac{\Delta_{0} R}{R}+\frac{\left|\nabla_{0} R\right|^{2}}{R^{2}}\right) .
\end{aligned}
$$

Combining this with Proposition 1, we thus have

$$
\operatorname{tr} \chi^{\prime}=\frac{2}{R}\left(1-\frac{\Delta_{0} R}{R}+\frac{\left|\nabla_{0} R\right|^{2}}{R^{2}}\right)-\frac{M_{0}}{R^{2}}
$$

in the limit $\delta \rightarrow 0$.

\section{Solutions to The Deformation Equation on $S_{0,0}$}

To prove our main Theorem 3 it suffices ${ }^{17}$ now to show that if $M_{0}$ verifies the the assumption (16), then an appropriate solution to the differential inequality on the standard sphere $S=S_{0,0}$,

$$
-\Delta R+R^{-1}|\nabla R|^{2}+R<2^{-1} M_{0},
$$

can be found. ${ }^{18}$

Let $R=e^{-\phi}$. Then the main deformation equation (39) reduces to

$$
\Delta \phi+1<\frac{1}{2} M_{0} e^{\phi}
$$

\footnotetext{
${ }^{17}$ Assuming $\delta \delta_{0}^{-1}, \delta_{0}$ sufficiently small, depending on $M_{0}$.

${ }^{18}$ From this point onwards, we drop the subscript ${ }_{0}$ in the connection coefficients as it will be clear from context that we consider the connection coefficients associated to the initial sphere $S_{0,0}$.
} 
We show below that (40) can be solved as long as $M_{0} \geq 0$ and $M_{0} \geq M_{*}>0$ on some open ball of $S$. Our approach provides an explicit construction using the Green's function for the Laplacian on $S$. The main observation is that given any function $\tilde{\phi}$, there exists a sufficiently large constant $C$ such that (40) is satisfied by $\phi=\tilde{\phi}+C$ on the set where $M_{0}$ has a positive lower bound. It is therefore sufficient first to construct a function $\tilde{\phi}$ satisfying (40) only on the complement of the set where $M_{0}$ has a positive lower bound. It turns out that an appropriately rescaled and cut-off version of the Green's function for the Laplacian satisfies this property.

We prove the following proposition, which together with Theorem 5, implies our main theorem (Theorems 3 and 4):

Proposition 4. Let $M_{*}=\min _{B_{p}(\epsilon)} M_{0}$. Then there exists a function $\phi_{\epsilon, M_{*}}$ verifying the inequality (40) and such that

$$
\begin{aligned}
\phi_{\epsilon, M_{*}} & \leq \log \left(\frac{1}{M_{*} \epsilon^{5}}\right)+O(1) \\
\left|\nabla \phi_{\epsilon, M_{*}}\right| & =O\left(\epsilon^{-1}\right), \quad\left|\nabla^{2} \phi_{\epsilon, M_{*}}\right|=O\left(\epsilon^{-2}\right) .
\end{aligned}
$$

3.1. Proof of the main theorem. Returning to our task of constructing a trapped surface, note that the upper bound (41) for $\phi$ corresponds to our desired lower bound for $R$. More precisely, (41) implies that for some $C>0$,

$$
\max _{S} \frac{1}{R} \leq e^{\max _{S} \phi} \leq \frac{C}{M_{*} \epsilon^{5}}
$$

In particular, for $M_{0}$ satisyfing the assumption of Theorem 4, i.e.,

$$
\inf _{B_{p}(\epsilon)} M_{0} \geq M_{*}>0
$$

the proposition implies the existence of a function $R$ verifying (39) and a lower bound $R>c_{0} M_{*} \epsilon^{5}$, for some constant $c_{0}$. Therefore, by Theorem 1 , given $M_{*}$ and $\epsilon$, we can choose $\delta$ sufficiently small such that the spacetime solution for the characteristic initial value problem remains smooth in $\mathcal{D}\left(u_{*}, \delta\right)$ for $u_{*}=1-r_{*}<1-c_{0} M_{*} \epsilon^{5}$. This guarantees that the sphere $\{(\underline{u}, u, \omega): \underline{u}=\delta, 1-u=R(\omega)\}$ lies within $\mathcal{D}\left(u_{*}, \delta\right)$. Moreover, given a function $R$ verifying (39), the term $C \delta_{0}$ in (20) can be made arbitrarily small by choosing $\delta$ small. Thus, by Theorem 5 , the sphere defined by $\{(\underline{u}, u, \omega): \underline{u}=\delta, 1-u=R(\omega)\}$ is a trapped surface in $\mathcal{D}\left(u_{*}, \delta\right)$. Since $R \geq r_{*}$, the constructed trapped surface has area at least $\gtrsim r_{*}^{2} \approx M_{*}^{2} \epsilon^{10}$.

This concludes the proof of the main theorem. It thus remains to prove Proposition 4.

3.2. Proof of Proposition 4. To this end, we use the following bounds for the Green's function of the standard sphere $S$. 
Lemma 2. Given a point $p$ in the standard unit sphere, define $\lambda$ to be the distance function from $p$. Then the function given by

$$
w=\sin \left(\log \left(\frac{\lambda}{2}\right)\right)
$$

satisfies

$$
\Delta_{d} w+\frac{1}{2}=2 \pi \delta_{p}
$$

where $\Delta_{d}$ is the distributional Laplacian on the standard sphere and $\delta_{p}$ is the Dirac measure at $p$. Moreover, $w$ obey the following bounds:

$$
w=\log \lambda+O(1), \quad|\nabla w|=O\left(\lambda^{-1}\right), \quad\left|\nabla^{2} w\right|=O\left(\lambda^{-2}\right) .
$$

Remark 5. In a more general setting where the metric $\gamma$ on $S$ is not the metric on the standard unit sphere, we can still use the Green's function to construct a desired solution to the elliptic inequality. More precisely, given a smooth riemannian metric $\gamma$ on $S$, there exists a function $w$, smooth outside the point $p$, such that

$$
\Delta_{d} w+\frac{1}{2 r_{0}}=2 \pi \delta_{p}
$$

where $\Delta_{d}$ is the distributional Laplacian associated to the metric $\gamma, \delta_{p}$ is the Dirac measure at $p$, and $r_{0}$ is defined by Area $(S)=4 \pi r_{0}$. Moreover, if $\lambda_{p}$ denotes the distance function from $p$,

$$
w=\chi \log \lambda_{p}+v
$$

with $v$ smooth in $S \backslash\{p\}$ and satisfying

$$
|v| \leq C, \quad|\nabla v|=o\left(\lambda^{-1}\right), \quad\left|\nabla^{2} v\right|=o\left(\lambda^{-2}\right) ;
$$

and $\chi$ a smooth cut-off function identically equals to 1 in a small neighborhood of $p$.

We refer the readers to Theorem 4.13 in [1] for a proof of this fact.

Using Lemma 2, we now proceed to the proof of Proposition 4:

Proof of Proposition 4. Consider the cut-off function

$$
\left\{\begin{array}{lll}
\chi_{\epsilon}=0 & \text { on } & B_{p}(\epsilon / 2) \\
\chi_{\epsilon}=1 & \text { on } & S \backslash B_{p}(\epsilon)
\end{array}\right.
$$


and define $\tilde{w}_{\epsilon}=\chi_{\epsilon} w+\left(1-\chi_{\epsilon}\right) \log \epsilon$. Note ${ }^{19}$ for that $\tilde{w}_{\epsilon}$ verifies the following properties:

$$
\left\{\begin{array}{lrr}
\tilde{w}_{\epsilon}=\log \epsilon, & \text { on } \quad B_{p}(\epsilon / 2) \\
\tilde{w}_{\epsilon}=\log \epsilon+O(1), & \text { on } \quad B_{p}(\epsilon) \backslash B_{p}(\epsilon / 2) \\
\tilde{w}_{\epsilon}=\log \lambda+O(1), & \text { on } \quad S \backslash B_{p}(\epsilon) \\
\nabla \tilde{w}_{\epsilon}=O\left(\epsilon^{-1}\right), & \text { on } \quad S \backslash B_{p}(\epsilon / 2) \\
\nabla^{2} \tilde{w}_{\epsilon}=O\left(\epsilon^{-2}\right), & \text { on } \quad S \backslash B_{p}(\epsilon / 2) \\
\Delta \tilde{w}_{\epsilon}+\frac{1}{2}=0, & \text { on } S \backslash B_{p}(\epsilon)
\end{array}\right.
$$

Consider now the function $\tilde{\phi}_{\epsilon}=3 \tilde{w}_{\epsilon}$ and observe that, on $S \backslash B_{p}(\epsilon)$, we must have,

$$
\Delta \tilde{\phi}_{\epsilon}+1=-\frac{3}{2}+1<0 \text {. }
$$

Thus, we have,

$$
\left\{\begin{array}{lrr}
\tilde{\phi}_{\epsilon}=3 \log \epsilon, & \text { on } \quad B_{p}(\epsilon / 2) \\
\tilde{\phi}_{\epsilon}=3 \log \epsilon+O(1), & & \text { on } \quad B_{p}(\epsilon) \backslash B_{p}(\epsilon / 2) \\
\tilde{\phi}_{\epsilon}=3 \log \lambda+O(1), & & \text { on } S \backslash B_{p}(\epsilon) \\
\nabla \tilde{\phi}_{\epsilon}=O\left(\epsilon^{-1}\right), & \text { on } \quad S \backslash B_{p}(\epsilon / 2) \\
\nabla^{2} \tilde{\phi}_{\epsilon}=O\left(\epsilon^{-2}\right), & \text { on } S \backslash B_{p}(\epsilon / 2) \\
\Delta \tilde{\phi}_{\epsilon}+1<0, & \text { on } S \backslash B_{p}(\epsilon)
\end{array}\right.
$$

Finally, we add a large constant to the function $\tilde{\phi}_{\epsilon}$ to obtain the desired solution to the inequality (40). More precisely, we let

$$
\phi_{\epsilon, M_{*}}=-\log s_{\epsilon, M_{*}}+\tilde{\phi}_{\epsilon},
$$

where $s_{\epsilon, M_{*}}$ is some small constant to be chosen later.

Clearly,

$$
\Delta \phi_{\epsilon, M_{*}}=\Delta \tilde{\phi}_{\epsilon}
$$

Therefore, on $S \backslash B_{p}(\epsilon)$,

$$
\Delta \phi_{\epsilon, M_{*}}+1=\Delta \tilde{\phi}_{\epsilon}+1<0 .
$$

By (47), there exists a positive constant $C_{0}^{\prime}$ such that, everywhere,

$$
\Delta \tilde{\phi}_{\epsilon} \leq \frac{C_{0}^{\prime}}{\epsilon^{2}}
$$

Thus, on $B_{p}(\epsilon)$,

$$
\Delta \phi_{\epsilon, M_{*}}+1=\Delta \tilde{\phi}_{\epsilon}+1<\frac{C_{0}}{\epsilon^{2}}
$$

\footnotetext{
${ }^{19}$ Note in particular the logarithmic cancellation in the formulas for $\nabla \tilde{w}_{\epsilon}$ and $\nabla^{2} \tilde{w}_{\epsilon}$.
} 
for some positive constant $C_{0}>0$. To ensure our inequality (40) we need to make sure that $\frac{C_{0}}{\epsilon^{2}}$ does not exceed $\frac{1}{2} M_{*} e^{\phi_{\epsilon, M_{*}}}$ on $B_{p}(\epsilon)$. We therefore need,

$$
\inf _{B_{p}(\epsilon)} \phi_{\epsilon, M_{*}} \geq \log \left(\frac{2 C_{0}}{M_{*} \epsilon^{2}}\right)
$$

Recall that (47) implies

$$
\inf _{B_{p}(\epsilon)} \phi_{\epsilon, M_{*}} \geq-\log s_{\epsilon, M_{*}}+3 \log \epsilon-\log C_{1}
$$

for some $C_{1}>0$. Therefore, it suffices to choose

$$
s_{\epsilon, M_{*}}=\frac{M_{*} \epsilon^{5}}{2 C_{0} C_{1}}
$$

and (40) is verified everywhere on $S$.

Finally, we use (47), (48) and (49) to check that $\phi_{\epsilon, M_{*}}$ obeys the bounds asserted in the proposition. By (47), we have the one-sided bound

$$
\phi_{\epsilon} \leq C,
$$

for some $C>0$. This, together with (48) and (49) implies

$$
\sup _{S} \phi_{\epsilon, M_{*}} \leq-\log \left(\frac{M_{*} \epsilon^{5}}{C_{0} C_{1}}\right)+O(1)=\log \left(\frac{1}{M_{*} \epsilon^{5}}\right)+O(1) .
$$

For the bounds for the first and second derivatives of $\phi_{\epsilon, M_{*}}$, notice that since $s_{\epsilon, M_{*}}$ is a constant, by (47), we have

$$
\begin{aligned}
\left|\nabla \phi_{\epsilon, M_{*}}\right| & =\left|\nabla \phi_{\epsilon}\right|=O\left(\epsilon^{-1}\right), \\
\left|\nabla^{2} \phi_{\epsilon, M_{*}}\right| & =\left|\nabla^{2} \phi_{\epsilon}\right|=O\left(\epsilon^{-2}\right) .
\end{aligned}
$$

\section{Acknowledgements}

We would like to thank Demetrios Christodoulou for his helpful suggestions on a previous version of the manuscript. We would also like to thank Pin Yu, Ovidiu Savin and Stefanos Aretakis for helpful remarks and useful discussions.

S Klainerman would like to thank the Fondation SMP for the support of this work. S. Klainerman is supported by the NSF grant DMS-0901250. J. Luk is supported by the NSF Postdoctoral Fellowship DMS-1204493. I. Rodnianski is supported by the NSF grant DMS-1001500. S. Klainerman and I. Rodnianski are also supported by the FRG grant DMS-1065710. 


\section{REFERENCES}

[1] T. Aubin, Non-linear Analysis on manifolds. Monge-Ampère equations., Springer-Verlag, 1982.

[2] D. Christodoulou, The formation of black holes in general relativity, Monographs in Mathematics, European Mathematical Soc. 2009.

[3] D. Christodoulou, S. Klainerman, The global nonlinear stability of the Minkowski space, Princeton mathematical series 41, 1993.

[4] S. Klainerman, F. Nicoló, The evolution problem in general relativity, Progress in mathematical physics, 2003.

[5] S. Klainerman, I. Rodnianski, On the formation of trapped surfaces, preprint 2009, to appear in Acta Mathematica.

[6] S. Klainerman, I. Rodnianski, On emerging of scarred surfaces for the Einstein vacuum equations, Discrete Contin. Dyn. Syst. 28 (2010), no. 3, 1007-1031.

[7] J. Luk, I. Rodnianski, Nonlinear interaction of impulsive gravitational waves for the vacuum Einstein equations, preprint, 2012, arXiv:1301.1072.

[8] M. Reiterer, E. Trubowitz Strongly focused gravitational waves. preprint 2009, arXiv:0906.3812

Department of Mathematics, Princeton University, Princeton NJ 08544

E-mail address: $\quad$ seri@math.princeton.edu

Department of Mathematics, University of Pennsylvania, Philadelphia PA 19104

E-mail address: jwluk@sas.upenn.edu

Department of Mathematics, Mit, Cambridge, Ma 02139

E-mail address: irod@math.mit.edu 